\title{
Concise review: The role of cancer-derived exosomes in tumorigenesis and immune cell modulation
}

\author{
Nhi Thao Huynh ${ }^{1,2}$, Khuong Duy Pham ${ }^{1,2}$, Nhat Chau Truong ${ }^{2,3, *}$
}

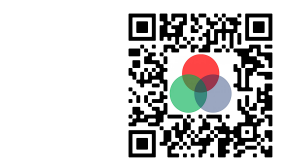

Use your smartphone to scan this QR code and download this article

\begin{abstract}
Exosomes are subcellular entities which were first discovered in the 1980s. Over the past decade scientists have discovered that they carry components of genetic information that allow for cell-cell communication and cell targeting. Exosomes secreted by cancer cells are termed cancer-derived exosomes (CDEs), and play an important role in tumor formation and progression. Specifically, CDEs mediate the communication between cancer cells, as well as between cancer cells and other cells in the tumor microenvironment, including cancer-associated fibroblasts, endothelial cells, mesenchymal stem cells, and effector immune cells. Additionally, through the vascular system and body fluids, CDEs can modulate response to drugs, increase angiogenesis, stimulate proliferation, promote invasion and metastasis, and facilitate escape from immune surveillance. This review will discuss the relationship between cancer cells and other cells (particularly immune cells), as mediated through CDEs, as well as the subsequent impact on tumorigenesis and immunomodulation. Understanding the role of CDEs in tumorigenesis and immune cell modulation will help advance their utilization in the diagnosis, prognosis, and treatment of cancer.
\end{abstract}

Key words: cancer, exosomes, extracellular vesicles, tumor microenvironment, tumorigenesis

\section{INTRODUCTION}

Exosomes are one of many subtypes of extracellular vesicles (EVs), along with microvesicles, apoptotic bodies, ectosomes, and oncosomes, among others. The diameter of exosomes ranges from 30 to $150 \mathrm{~nm}$. Exosomes are released, by almost every type of cell (including normal and abnormal), into the extracellular space through the fusion between the multi-vesicle bodies (MVBs) and the plasma membrane ${ }^{1}$. They can be found in a wide variety of body fluids, including blood, saliva, and breast milk. This indicates that one of their potential functions is modulating physiological responses ${ }^{2}$.

In the 1980s, nano-sized vesicles were first discovered in extracellular space and termed "exosomes" ${ }^{3}$. At the time, exosomes were only known to carry cellular waste (homeostasis by-products) and dispose them to the extracellular space. Thus, they were not regarded as having any significant effects on other cells. Over time, however, more discoveries on exosomes emerged; they were no longer regarded as just cellular waste. Today, they are known to transport different types of genetic and molecular cargoes (including lipids, proteins, nucleic acids and metabolites) between cells to facilitate communication and to impact the function and behavior of recipient/target cells ${ }^{4}$.

\section{Exosome biogenesis}

The biogenesis of exosomes involves an initial inward budding of the cell membrane to form early endosomes. The early endosomal membrane then invaginates inwardly to form intraluminal vesicles (ILVs) in the lumen. As the early endosomes mature into late endosomes, this facilitates the formation of multivesicular bodies (MVBs). Next, MVBs are either degraded when fusing with lysosomes or secreted into the extracellular space through fusion with the plasma membrane. Exosome biogenesis is modulated by several pathways, including the endosomal sorting complexes required for transport (ESCRT)dependent and ESCRT-independent pathways. The ESCRT is comprised of four sub-complexes, which include ESCRT-0 to -III. The two signaling pathways promote the maturation of endosomes and formation of MVBs, which are responsible for cargo sorting. Exocytosis and secretion of exosomes require the Rab-dependent trafficking pathways, which include Rab11, Rab27, and Rab35. Also, other factors contribute to ILV formation, including the p53/TSAP6 pathway, tetraspanin CD63, specific glycan modification, and/or mechanisms that depend on lipids ${ }^{5-7}$.

\section{Exosomal components}

Exosomes are referred to as packages or envelopes that mediate cell-to-cell communication. They facil-

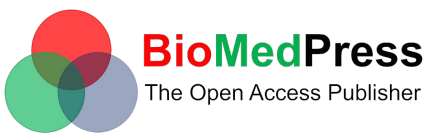


itate the communication between normal cells, between cancer cells, but also between cancer cells and other cells in the tumor microenvironment (TME), including mesenchymal stem cells (MSCs), normal fibroblasts, and cancer-associated fibroblasts (CAFs). Indeed, the signals emitted by cancer cells vary greatly depending on the type of cells they interact with. Cancer-derived exosomes (CDEs) contain genetic and molecular components, in the form of mRNA , miRNAs, proteins, lipids, metabolites, and nucleic acids ${ }^{8}$. The cancer-derived exosomal components may carry similar genetic information as the parental cells but also may have their own characteristics, contributing to the diversity in the tumor development process. Thus, exosomes secreted from cancer cells may carry and transmit components that reflect the metabolic state and function of parental cells, but may also contain other components, including other EVs like oncosomes ${ }^{9,10}$. Indeed, components in CDEs are mostly responsible for mediating tumor growth ${ }^{11}$. A regularly updated database of exosome components can be found at this site (http://www.exocarta.org).

\section{EXOSOMES IN CELL-CELL COMMUNICATION}

Communication between cells can sustain homeostasis in the body but can also manifest in disease. In addition to notable communication mechanisms, including direct cell-cell interactions or uptake of multiple extracellular signaling molecules (e.g. cytokines, hormones, and growth factors), exosomes have emerged as extracellular signaling molecules critical for both inter- and intracellular communications $^{2}$. Importantly, exosomes help to maintain the body's homeostasis, mediate cell signals, and modulate immune responses. However, they can also contribute to the pathogenesis of diseases and disorders. For instance, they can promote neurodegeneration in neural disorders, as well as tumorigenesis, progression, and metastasis in cancer.

During cell-cell communication, exosomes from cells can transport messages to various designated sites. This process can be mediated by different proteins, such as Rab protein and tetraspanin. CDEs are secreted by cancer cells and attach to nearby cells or to the extracellular matrix (ECM), or can float along the bloodstream and body fluids. When the exosomes reach their destinations, they can be taken up by recipient cells through endocytosis, via fusion with membrane or internalized by receptors ${ }^{6}$.

\section{Effects of cancer-derived exosomes on var-} ious cells

As mentioned above, exosomes are secreted by many types of cells, including cancer cells. CDEs carry a collection of bioactive molecules, such as lipids, proteins, nucleic acids, and nucleic acid components (noncoding RNA, mRNA, and DNA fragments). These components participate in a variety of cellular activities, including intracellular communication, chemotherapy resistance, angiogenesis, modulation of TME, immune reaction mediation, stimulation of cell invasion, and metastasis. Specifically, CDEs affect cancer progression and metastasis in four different processes: first, they promote angiogenesis for cancer proliferation; second, they modulate cancer migration and invasion; third, they induce cancer cells to escape immune system attack; and last, they prime cancer metastasis via promoting the formation of premetastatic niches $^{12}$ (Figure 1).

\section{Effects of CDEs on communication between cancer cells}

For cancer to progress, a crucial step is proliferation of cancer cells, which is mediated mainly by soluble growth factors. To facilitate proliferation, cancer cells must communicate with each other in the TME through intricate signaling pathways which rely on exosomes to carry and transmit bioactive signaling molecules to adjacent and distant cells. Indeed, it has been shown in several research studies that CDEs are capable of promoting tumor proliferation and progression via these signaling mechanisms ${ }^{13}$. According to a study published in 2015 by Raimondo et al., exosomes released from chronic myeloid leukemia (CML) can also induce an autocrine effect, promoting the growth and survival of cancer cells.

Other examples of the role of CDEs in cancer proliferation include exosomal CD97 from gastric cancer (GC), which mediates cell proliferation and invasion of GC cells by activating the mitogen-activated protein kinase (MAPK) pathway, and exosomal miRNAs, which activate the CD97-associated pathway ${ }^{1}$. Furthermore, exosomes derived from GC activate several pathways, including the PI3K/Akt and MAPK/ERK (extracellular-regulated protein kinase), to promote tumor proliferation ${ }^{14}$. In 2017, Pan et al. concluded in their research that ZFAS1, a long non-coding RNA (LncRNA), which can be found in GC-derived exosomes, was capable of promoting tumor proliferation and metastasis by mediating the cell cycle process and epithelial-mesenchymal transition (EMT) ${ }^{15}$.

Another function of CDEs is they can regulate the migratory status of cancer cells. Secreted CDEs can be 

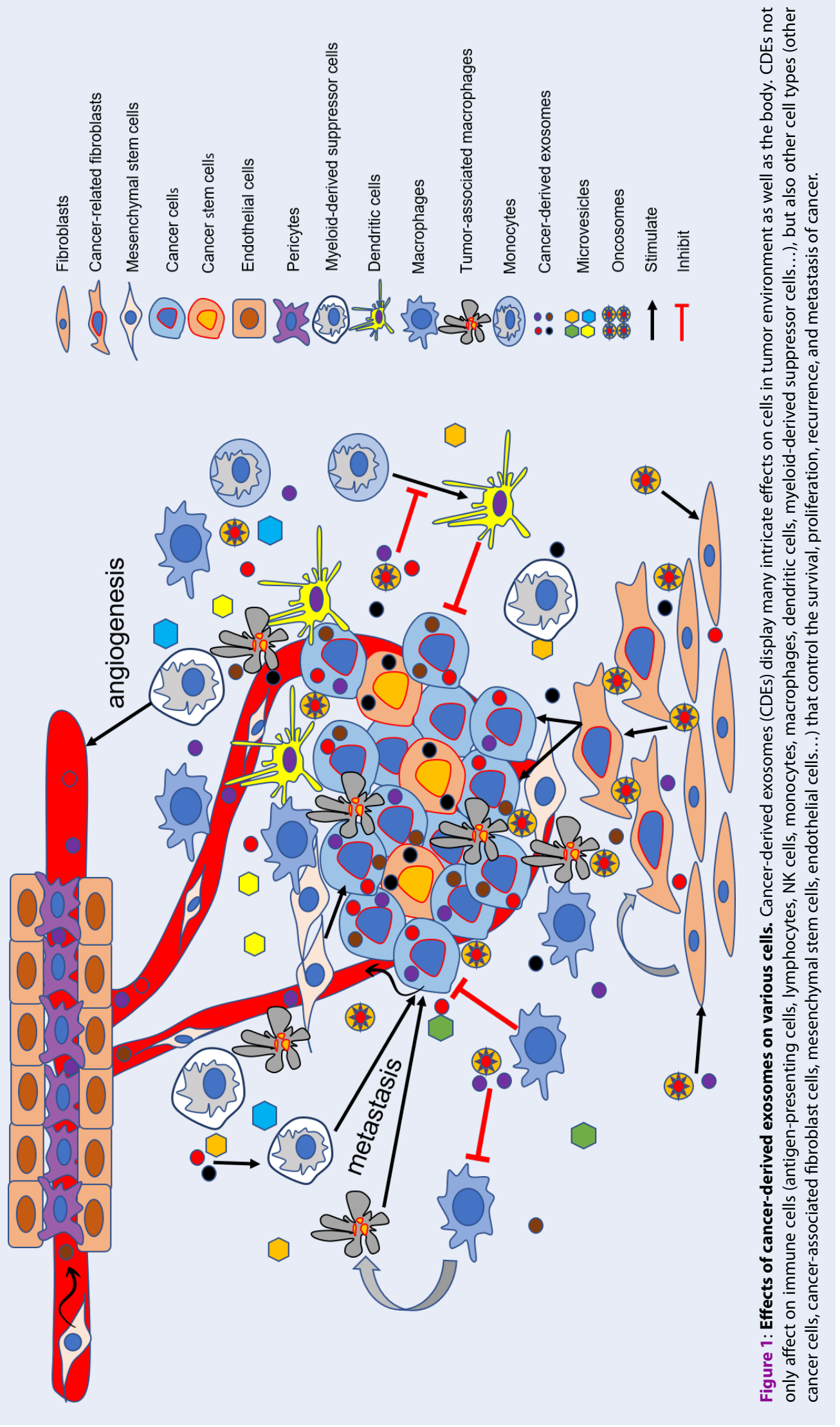
taken up by adjacent cancer cells, and can act upon those cells. For instance, exosomes secreted by hypoxic prostate cancer carry a larger number of proteins, such as adherent junction molecules. The CDEs rich in these kinds of proteins can potentially stimulate invasiveness, stemness, and motility of naive prostate cancer cells ${ }^{16}$.

Besides their involvement in tumorigenesis, CDEs can also promote chemoresistance through cell-cell communication of malignant cells. Docetaxel is a chemotherapy medication that is used to treat several types of cancers. In 2012, Corcoran et al. confirmed after studying docetaxel resistance in prostate cancer (PC) patients that CDEs derived from PC are partially responsible for docetaxel resistance in secondary cells. Moreover, in lung cancer, cisplatin resistance can be transferred from donor cells to recipient cells by lung cancer-derived exosomes that expressed a low level of miR-100-5p; this led to mTOR being affected, thereby impacting and regulating cellular reaction to cisplatin in the recipient cells ${ }^{17}$.

\section{Effect of CDEs on CAFs}

Cancer-associated fibroblast cells (CAFs) are diverse and heterogeneous ${ }^{18}$. It is thought that CAFs are transformed by cancer cells to support the growth of the cancer, in general, and to modulate tumor progression, in particular. CAFs make up the bulk of the tumor, and they are capable of activating the F19+ positive fibroblast population in the malignant proliferative fibroblast pool ${ }^{19}$. Studies showed that altered p53 function in CAFs contributes to tumor progression ${ }^{20}$. Another study showed that IL-6 secreted from CAFs depletes p53 through STAT3 in prostate cancer cells $^{21}$. Therefore, CAFs serve as a powerful partner for cancer cells, enabling the latter to invade and metastasize to many sites. For the sake of simplicity, CAFs can originate from many different types of cells, such as fibroblasts ${ }^{22}$, or from the transformation of $\mathrm{MSCs}^{23}$, fat cells ${ }^{24}$, bone marrow stem cells ${ }^{25}$. They can migrate to the tumor to assist with immune modulation, such as reducing inflammation by secreting cytokines.

CAFs were originally normal fibroblasts but became altered by cancer cells. In the TME, these cells appear to be transformed into cancer-associated- or tumor growth-helper cells, changing their metabolic reprogramming to create high fuel sources like ketones and lactates. These fuel tanks were then delivered to cancer cells through monocarboxylate (MCT) transporters or exosomes to support cancer cell proliferation $^{26}$. These findings showed that cancer cells benefit from both phenotypic heterogeneous features and diverse metabolic patterns. A study on a breast cancer cell line showed that MiR-122 secreted from secretory vesicles could facilitate reprogramming of glucose metabolism and promote metastasis ${ }^{27}$. A study of exosomes secreted from cancer cells showed stimulation of fibroblasts into myofibroblast through tumor growth factor (TGF)- $\beta$, thereby contributing to tumor progression ${ }^{28}$. In another study, the increase in LINC00355 expression of CAFs promoted the proliferation and invasiveness of bladder cancer ${ }^{29}$. In a study of ovarian cancer, it was found that exosomes from ovarian cancer cells affected fibroblast cells of tumor origin and cells adjacent to the tumor ${ }^{30}$. Exosome secretion of CAFs also induced resistance in a study of pancreatic carcinoma ${ }^{31}$. Resistance to exosomes secreted by CAFs has also been observed in a study of colorectal cancer (CRC) ${ }^{32}$. The interaction between cancer cells and CAFs appears to promote tumor progression in a variety of ways, of which communication via exosomes is highly predominant.

\section{Effects of CDEs in cancer cell-endothelial cell communication}

Endothelial cells (ECs) play many roles in the regulation of homeostasis. First, they manage the passage of nutrients and oxygen; second, they represent a variety of trophic factors; and third, they dispose metabolic by-products. Cancer cells, similar to normal cells, also need nutrients, oxygen, and growth factors to survive, proliferate, and metastasize. On this basis, exosomes are considered to be important in the communication between cancer cells and ECs ${ }^{13,33}$. For instance, exosomes derived from CML (LAMA84) affect the signal transduction pathways in ECs and activate angiogenesis through the release of IL- ${ }^{34}$. According to Thompson et al., in a 2013 study, when the expression of enzyme heparanase in human cancer cells (e.g. breast cancer and myeloma) was increased, the secretion of exosomes was also increased. In cells with higher expression of heparanase, the exosomes also carried higher levels of syndecan-1, hepatocyte growth factor, and vascular endothelial growth factor (VEGF), which promoted tumor migration on fibronectin and $\mathrm{EC}$ invasion through the $\mathrm{ECM}^{35}$. Another example is angiogenesis promotion in epithelial ovarian cancer (EOC) cells via metastasis-associated lung adenocarcinoma transcript 1 (MALAT1)-rich exosomes. EOC-derived exosomes transfer MALAT1 to human umbilical vein ECs (HUVECs), activating the expression of the angiogenesis-related gene and eventually promoting angiogenesis ${ }^{36}$.

CDEs can also promote cancer metastasis by affecting vascular integrity ${ }^{13}$. For example, in ECs, CDEs 
which carry the miR-105 cause destruction on the tight junction and destroy the integrity of the vascular barriers against metastasis. Moreover, miR-105 overexpression in non-metastatic cancer cells promotes metastasis and causes blood vessels in distant organs to become more permeable ${ }^{37}$. Glioblastoma multiforme (GBM)-derived exosomes enriched with VEGF-A promote angiogenesis and the permeable potential of human brain ECs in vitro ${ }^{38}$. In addition, exosomes that are secreted from cancer cells under hypoxic conditions can play dual roles in both tumor progression and metastasis ${ }^{39}$. In ex vivo and in vitro settings, GBM-derived exosomes developed under hypoxic conditions (in comparison with normoxic conditions) promote angiogenesis by phenotypic alteration of ECs. Moreover, in the same settings, ECs were primed to release multiple growth factors and cytokines, to activate late pericytes via $\mathrm{PI} 3 \mathrm{~K} / \mathrm{AKT}$, and to promote migration ${ }^{40}$. In CRC, exosomes secreted under hypoxic conditions stimulated ECs to proliferate and migrate. Furthermore, hypoxic CRC-derived exosomes loaded with Wnt4 were able to augment $\beta$-catenin nuclear translocation signals in ECs, prompting the ECs to proliferate and migrate ${ }^{41}$.

\section{Effects of exosomes from MSCs on cancer cells}

The potential of MSCs for use in the treatment of a wide variety of diseases is highly attractive and, thus, has garnered great interest from scientists ${ }^{42-46}$. The interaction between cancer cells and MSCs is also of particular interest to many researchers, whether the interaction is mediated by MSC-derived exosomes acting on cancer cells or by CDEs acting on MSCs. Many studies show that exosomes secreted from MSC promote tumor growth ${ }^{47,48}$. However, there is also research which suggests that exosomes secreted from MSC do not promote the proliferation of cancer cells in vitro, but are more prominent in their regenerative ability ${ }^{49}$. Another study in mouth squamous cell carcinoma showed that exosomes from stem cells help inhibit angiogenesis and tumors ${ }^{50}$. Also, a study on HepG2 liver cancer cell line showed that exosomes from bone marrow MSCs inhibited tumor growth both in vitro and in viv $o^{51}$. Exosomes from adipose MSCs also inhibited the proliferation of hepatocellular carcinoma (HCC) in mice ${ }^{52}$. Alzahrani et al. (2018) showed that exosomes from cancer stem cells (CSCs) induce and promote tumor progression in HCC while MSC-secreted exosomes have an inhibitory effect ${ }^{53}$. Indeed, the diversity of exosomes in many different cell lines has led researchers to further investigate the possible use of exosomes in cancer treatment.

\section{CDEs in cancer cell-immune cell communi-} cation

According to the hallmarks of cancer, malignant cells can devise multiple ways to avoid being destroyed by the different arms of the immune system ${ }^{54}$. In order to survive and proliferate, tumors need to avoid being detected by either inhibiting antigen-presenting cells (APCs) or cytotoxic T lymphocyte (CTL) function, or enhancing immune suppressor cells to potentially shut down the immune system. Besides immunosuppression, the immune system can be reversely modulated to assist in the development and survival of cancer cells. CDEs contribute to each of these strategies, via proteins on their surface, or nucleic acids, or intra-vesicle cytokines ${ }^{55}$. CDEs prevent the immune system from performing its antitumor activities. They do so by interfering with the differentiation and activation of immune suppressor genes, obstructing immune cell proliferation, altering antigen presentation, promoting activated T-cell apoptosis, reducing the activity of natural killer (NK) ${ }^{56}$ cells, and affecting monocyte differentiation ${ }^{57}$. CDEs also hamper with the immune surveillance of the host by elevating signals released by tumor cells; these signals include inflammation, tumorigenesis, and immune escape of cancer cells. Moreover, CDEs can modulate immune responses by outsmarting stromal and immune cells. Thus, eventually, CDEs act as crucial contributors in regulating immunoediting not only at primary tumor sites but also at secondary sites ${ }^{13}$.

\section{Monocytes}

Monocyte account for $5 \%$ of circulating leukocytes (white blood cells) in most mammalian species ${ }^{58}$. Monocytes are sensitive to changes in the environment which make them plastic and heterogeneous. They are able to alter their functional phenotype with regards to different stimuli in the environment, such as growth factors, cytokines, and microbial products. Moreover, they can differentiate into either macrophages or dendritic cells. Therefore, they are crucial for both innate and adaptive immune responses ${ }^{59-61}$. Initially, the immune system was only known for its ability to protect the human body from infections and cancer development, via mechanisms like immune surveillance. However, recent studies have demonstrated that some unresolved immune activities, such as chronic inflammation, can induce cancer progression and metastasis ${ }^{62,63}$. Moreover, tumor cells and their TME can secrete molecules that affect monocytes in different ways, including impacting their function, migration, recruitment, and differentiation $^{31}$. For instance, chronic lymphocytic 
leukemia (CLL)-derived exosomes were taken up by monocytes, which then activated the expression of programmed death-ligand 1 (PD-L1), a molecule that can suppress the immune responses and trigger the redirection of both monocytes and macrophages towards pro-tumorigenic phenotypes. Moreover, this triggered the release of cytokines, including C-C motif chemokine ligand (CCL)-2, CCL-4, and interleukin (IL)-6, which in turn stimulated nuclear factor$\mathrm{kB}(\mathrm{NF}-\mathrm{kB})$ activity ${ }^{56}$. Additionally, NF-kB is commonly known for its contribution to immune activation and inflammation. However, data suggest that constitutively stimulated NF-kB is involved in tumorigenesis by promoting cancer proliferation, angiogenesis, and metastasis, while inhibiting apoptosis ${ }^{64}$. Exosomes derived from malignant ascites of ovarian cancer cells were examined for their ability to regulate monocytic biological functions. The CDEs were taken up by THP-1 monocytic cells via cell surface receptors, Toll-like receptor (TLR)-2 and TLR4 , which then triggered the production of multiple cytokines, including IL-1B, IL-6, and tumor necrosis factor (TNF)-a, and also activated NF-kB. Moreover, CDEs can also activate the signal transducer and activator of transcription 3 (STAT3) pathways via the release of IL-6 in an autocrine/paracrine manner ${ }^{65}$. Furthermore, activated NF-kB and STAT3 interact with each other to modulate communication between tumor cells and the TME, which consists of inflammatory cells from the immune system that respond to the cancer cells. Therefore, NF-kB and STAT3, when combined, play important roles in the regulation of cytokines to induce tumorigenesis, and regulate angiogenesis, metastasis, and cancer cell apoptosis ${ }^{66}$.

\section{Macrophages}

Macrophages have specialized functions in the immune system, with the main function being to recognize, engulf, and destroy foreign or harmful factors, such as bacteria and pathogens. Moreover, macrophages can also present antigens to $\mathrm{T}$ cells or promote inflammation through the release of molecules, which then activate the immune responses. One of special features of macrophages is that they can be phenotypically-polarized, depending on specific requirements from the environment. They can be polarized into two main subtypes: classical activation (M1 cells) or alternative activation (M2 cells) ${ }^{67}$. The activation of both M1 and M2 macrophages could potentially achieve opposite outcomes. The activation of M1 macrophages increasingly produces pro-inflammatory molecules, such as cytokines and chemokines, which induce the elimination of foreign factors and infections. Activated M2 macrophages release anti-inflammatory signals and induce tumor progression, promote angiogenesis, and heal wounds. Several studies had shown that exosomes participate in the communication between cancer cells and tumor-associated macrophages (TAMs), thereby modulating how these macrophages are polarized and will function ${ }^{68}$. For example, in CRC, CDEs play an important and active role in determining that the macrophages will be polarized into a more cancer-favorable, i.e. pro-tumorigenic phenotype, by utilizing the cytoskeleton-centric proteins as a functional unit ${ }^{69}$. Breast CDEs can induce a proinflammatory response in M1 macrophages by activating NF-kB signaling which leads to the production of inflammatory cytokines in monocytes. Moreover, only exosomes secreted by cancer cell lines can stimulate macrophages. TLR-2 interacts with proteins on the CDE surface to induce an inflammatory response by the stimulated macrophages. Also, the communication between breast CDEs and macrophages, combined with the existence of TLR-2 on macrophages and palmitoylated protein ligands on CDEs, can activate $\mathrm{NF}-\mathrm{kB}^{70}$.

\section{Dendritic cells (DCs)}

Dendritic cells (DCs) serve as antigen-presenting cells (APCs) in the immune system, and play pivotal roles in the primary immune activation. DCs are important to both innate and adaptive immunity since they can communicate with and regulate the responses of innate immune cells. Moreover, DCs are considered to be professional APCs because they are the only APCs that can initiate the response of naive T lymphocytes, which makes them crucial to the acquired immune stimulation ${ }^{71}$. Since DCs contribute significantly to the immune defense mechanisms, cancer cells have interfered with DC formation, migration, and function. Moreover, cancer cells also enhance the production of immature DCs, and these cells potentially become immune suppressors ${ }^{72}$. In 2007, Yu et al. concluded that CDEs are immune suppressive through the inhibition of DC differentiation. In murine models, $\mathrm{CD}_{1} 1^{+}$myeloid precursor cells in bone marrow interact with CDEs in vitro and stimulate the secretion of IL- 6 , which then partially stops these precursor cells from differentiating into DCs. Moreover, in the human breast cancer cell line MDAMB-231, CDEs were also shown to obstruct DC differentiation. These examples demonstrate that the inhibition of DC differentiation by CDEs contributes 
greatly to the immune escape of cancer cells ${ }^{73}$. CDEs can downregulate TLR-4 expression by transporting miR-203 to DCs, subsequently decreasing the production of TNF- $\alpha$ and IL-12, and suppressing DCinduced immune responses ${ }^{37}$.

\section{Myeloid-derived suppressor cells (MDSCs)}

Myeloid cells (or myelocytes) originate in the bone marrow and are comprised of different types of immune cells, such as mononuclear myelocytes (monocytes, macrophages, and dendritic cells) and granulocytic myelocytes (neutrophils, eosinophils, basophils, and mast cells). Myeloid cells are generated in response to stimuli from pathogenic factors and create a defense barrier to protect the host from infections. During tumor growth, myelocytes are differentiated into myeloid-derived suppressor cells (MDSCs), which contribute significantly to cancer progression by inducing cancer survival, promoting angiogenesis, promoting invasion of healthy cells, and promoting metastasis of cancer cells; thus, MDSCs are important contributors in the $\mathrm{TME}^{74,75}$. Cancer-derived exosomes, when internalized by myeloid cells, can induce these cells to alter their differentiation pathway to MDSCs $\left(\mathrm{CD} 11 \mathrm{~b}^{+} \mathrm{Gr}-1^{+}\right)$. The differentiated cells carry traits and functions of MDSCs, such as induction of tumor progression. Moreover, in vivo MDSCs can induce tumor development via CDEs enriched with prostaglandin E2 (PGE2) and TGF- $\beta$. Both PGE2 and TGF- $\beta$ are molecules that can suppress immune responses and promote angiogenesis by triggering the production of several pro-inflammatory cytokines, such as Cox 2 and IL-6. Moreover, these two molecules also produce and elevate the activity of VEGF, which in turn induce tumor migration and metastasis in the late stages of cancer ${ }^{76}$.

In addition, MDSCs internalized CDEs that are enriched with proteins Hsp70 and Hsp72, and then trigger an autocrine release of pro-inflammatory cytokine IL-6, thereby stimulating the phosphorylation of STAT3 in a TL2/MyD88-dependent fashion and facilitating expansion of MDSCs. This subsequently suppresses immune surveillance and anti-tumor immunity ${ }^{77,78}$.

On the other hand, many studies have shown that exosomes secreted from cancer cells suppress the immunity of immune cells, such as $\mathrm{T}$ cells and macrophage cells ${ }^{68,79}$. They can also turn into tumorassociated macrophages (TAM), which facilitate accelerated metastasis ${ }^{80-82}$. Cancer cells attack immune cells with exosomes carrying PDL-1 to T cells via $\mathrm{PD}-1$ receptors ${ }^{83}$. The immunosuppressive capacity of melanoma-derived exosomes has also been noted $^{84}$. Exosomes secreted from gastric cancer cells alter the cycle of T CD8 cells and alter gene expression of specific T CD8 to increase expression of immunosuppressive genes such as FOXP3 and IL-10 ${ }^{85}$. Exosomes expressing miR-940 secreted from ovarian carcinoma cells induce macrophages to switch to the TAM phenotype ${ }^{80}$. TAMs were also expressed after interacting with exosomes secreted from glioblastoma (GBM)-derived stem cells (GSCs) ${ }^{86}$. Exosome RPPH1 secreted from CRC also promotes the TAM phenotype to promote metastasis and CRC cell proliferation ${ }^{81}$. It can be seen that in a variety of ways exosomes derived from cancer cells can induce immune cells to help them spread faster. Through the exosomes, they can also rapidly destroy and inhibit immune cells through markers such as PD-1, CTLA4. The targeting and blocking of biological markers on these exosomes to treat cancer has been and is being studied by many immunological studies.

\section{APPLICATION OF CANCER-DERIVED EXOSOMES (CDES)}

Exosomes derived from cancer cells promote cancer development at different stages by transporting certain bioactive molecules that can induce changes in the TME to influence nearby cells or cells at distant sites. Since CDEs participate in the interaction between cancer and non-cancer cells, and they also possess some traits from the parental cells, this suggests that CDEs can potentially be used as biomarkers for cancer diagnosis, prognosis, and therapy ${ }^{87}$. Besides, it has been shown that CDEs can assist cancer cells to avoid attack from the immune system, and can contribute to therapeutic resistance in cancer patients ${ }^{88}$. Thus, an in-depth understanding of these mechanisms could equip us with information on how to improve cancer therapies. Still, the greatest advantage of CDEs in clinical applications is the utilization of their membrane structures, which are similar to other cells in the body, to transport anti-cancer drugs; thus, they have promising potential to be efficient cancer-targeted drug delivery vehicles ${ }^{2}$.

\section{CDEs as potential biomarkers for diagnosis and prognosis}

It has been shown that the contents of CDEs are similar, sometimes identical, to the intracellular conditions of their parental cells. Therefore, it was suggested that real-time observation of alterations inside CDEs could provide pertinent information about cancer cells. Moreover, since exosomes circulate within body fluids, they can be considered as liquid biopsies, 
which have been proven to be more convenient compared to tissue biopsies as they are less invasive, easily collected, fast, and more economical ${ }^{2}$. Biomarkers are biological indicators, usually in the form of a protein or protein fragment, that can be recognized in the patient's fluids (blood or urine), but not in healthy individuals ${ }^{89}$. Biomarkers are commonly used in detecting early stages of diseases or recurring diseases and assessing patient survival and recovery possibilities. Early detection of cancer could improve the odds of successful treatment and subsequently, elevate the patient's chances of survival. Thus, CDEs could potentially serve as biomarkers in cancer diagnosis, as well as providing information to guide cancer prognosis and monitoring.

The role of exosomes in cancer treatment is undeniably important. Yet, many areas remain to be investigated, such as the optimal conditions in which exosomes should be stored. In 2013, exosomes derived from blood plasma were assessed for their ability to remain stable under different storage conditions. After 90 days, the exosomes were analyzed via Western blot using TSG101, a marker for exosomes. It was demonstrated that the stability of the exosomes was still intact. Furthermore, the exosomes were still able to be internalized by the target cells, indicating that they were still active in the biological sense ${ }^{90}$. One important consideration is that plasma collected from cancer patients tends to produce more isolated exosomes than those from healthy subjects. Moreover, exosomes from cancer patients can strongly induce immune suppression, as was shown by the downregulation of CD69 expression on $\mathrm{CD}^{+}{ }^{+} \mathrm{T}$ effector lymphocytes $^{91}$.

As mentioned above, CDEs can create a suitable TME to promote cancer metastasis, via stimulation of specific patterns of metastasis. Moreover, CDEs carry certain integrins that can regulate cancer metastasis by directing the cancer cells to specific destinations. For instance, CDEs that carry integrin ITG $\alpha 6$ $\beta 4$ and ITG $\alpha 6-\beta 1$ can connect with lung fibroblasts and epithelial cells, leading to lung tropism; these cancer cells may prefer to metastasize to the lung. Meanwhile, CDEs enriched with ITG $\alpha \mathrm{v}-\beta 5$ can adhere to Kupffer cells and skew metastasis to the liver. This phenomenon demonstrated that integrins, which are considered to be the first bioactive molecules, can forecast which specific organs the cancer cells might metastasize to. Therefore, CDEs enriched with integrins could potentially be biomarkers for cancer organotropism ${ }^{92}$. In addition, exosomes derived from advanced melanoma have been found to transport proteins that are considered to have a "melanoma signature", such as tyrosinaserelated protein-2 (TYRP2), very late antigen-4 (VLA4), MET, HSP70, and HSP90 isoform. This signature can dictate the site of metastasis. Not only that, increased levels of exosomal TYRP2 and MET, combined with increased levels of other proteins in the exosomes, can also indicate cancer progression ${ }^{93}$. In head and neck squamous cell carcinomas (HNSCC), the overexpression of $\mathrm{CD} 44 \mathrm{v} 3$, a protein that is associated with tumor and is transported by CDEs, can indicate the status of HNSCC, including tumor progression and metastasis. Additionally, CDEs enriched with CD44v3 can suppress immune responses and act as an indicator of advanced disease stage and lymph node metastasis. Therefore, CD44v3 ${ }^{(+)}$CDEs can serve as a biomarker in the diagnosis and prognosis of $\mathrm{HNSCC}^{94}$.

\section{CDEs as vehicles of drug therapy}

Based on the current understanding of exosome, several studies have developed cancer screening diagnostics using signature markers of exosomes. Exosomal RPPH1 has been suggested as a diagnostic biomarker of $\mathrm{CRC}^{81}$. Glypican-1 (GPC1) in exosome secreted from pancreatic cancer cells can also be used in diagnosis $^{95}$. Additionally, exosomes have been used in diagnosing non-small cell lung cancer (NSCLC) ${ }^{96}$. A major advantage of exosomes is that cancer screening and diagnosis can be tested using only blood or urine, as opposed to tumor biopsies. Given the small size of exosomes, many researchers have focused on their use as a nanoscale targeted drug delivery system (of small molecular drugs) for the treatment of many diseases, including cancer, to promote long-term effects.

\section{CONCLUSION AND PERSPECTIVE}

In conclusion, exosomes are carriers that transport varied types of constituents from one cell to another, inducing target cells to alter in phenotype and function. Exosomes play a pivotal role in facilitating cellcell communication. Indeed, they are found in most cell types, including cancer cells. When cancer cells interact with other cancer cells, via CDEs, this can cause cancer cells to promote a more tumorigenic environment. Thus, CDEs can change the TME, support MET and EMT processes, stimulate cancer progression, and augment metastasis. Research studies on CDEs have demonstrated that CDEs, due to unique cargoes they transport, can potentially serve as biomarkers in cancer diagnosis and prognosis. Moreover, CDEs can play a pivotal role in cancer therapy 
since they can be curated to specifically target cancer cells through cell signaling. The use of exosomes to deliver drugs for cancer therapy can be less invasive and more physiologically relevant since they have similar structures with other cells in the TME. More extensive studies on exosomes are needed, such as further investigations into the mechanisms of transport and cell-cell communication mediated by CDEs. Nevertheless, a greater understanding of exosomes will provide greater insight and perspective on how to optimize the use of CDEs to treat different types of cancer.

\section{ABBREVIATIONS}

APCs: Antigen-presenting cells

CAFs: Cancer-associated fibroblast cells

CDEs: Cancer-derived exosomes

CLL: Chronic lymphocytic leukemia

CML: Chronic myeloid leukemia

CRC: Colorectal cancer

CTL: Cytotoxic T lymphocyte

DCs: Dendritic cells

ECM: Extracellular matrix

ECs: Endothelial cells

EOC: Epithelial ovarian cancer

ESCRT: Endosomal sorting complexes required for transport

EVs: Extracellular vesicles

GBM: Glioblastoma multiforme

GC: Gastric cancer

HUVECs: Human umbilical vein endothelial cells

ILVs: Intraluminal vesicles

LncRNA: Long non-coding RNA

MALAT1: Metastasis-associated lung adenocarcinoma transcript 1

MAPK: Mitogen-activated protein kinase

MAPK/ERK: Extracellular-regulated protein kinase

MDSCs: Myeloid-derived suppressor cells

MSCs: Mesenchymal stem cells

MVBs: Multivesicular bodies

NF-kB: Nuclear factor kb

NK: Natural killer

PC: Prostate cancer

PD-L1: Programmed death-ligand 1

PGE2: Prostaglandin E2

STAT3: Signal transducer and activator of transcription 3

TAMs: Tumor-associated macrophages

TLR: Toll-like receptor

TME: Tumor microenvironment

TNF: Tumor necrosis factor

VEGF: Vascular endothelial growth factor

\section{ACKNOWLEDGMENTS}

None.

\section{AUTHOR'S CONTRIBUTIONS}

All authors equally contributed to this work. All authors read and approved the final manuscript.

\section{AVAILABILITY OF DATA AND MATERIALS}

Not applicable.

\section{ETHICS APPROVAL AND CONSENT TO PARTICIPATE}

Not applicable.

\section{CONSENT FOR PUBLICATION}

Not applicable.

\section{COMPETING INTERESTS}

The authors declare that they have no competing interests.

\section{REFERENCES}

1. Li X, et al. Challenges and opportunities in exosome researchPerspectives from biology, engineering, and cancer therapy. APL Bioeng. 2019;3(1):011503. PMID: 31069333. Available from: .https://doi.org/10.1063/1.5087122.

2. Tai $\mathrm{YL}$, et al. Exosomes in cancer development and clinical applications. Cancer Sci. 2018;109(8):2364-2374. PMID: 29908100. Available from: https://doi.org/10.1111/cas.13697.

3. Johnstone RM, et al. Vesicle formation during reticulocyte maturation. Association of plasma membrane activities with released vesicles (exosomes). J Biol Chem. 1987;262(19):9412-9420.

4. Zhang $X$, et al. The function of tumor-derived exosomes. J BUON. 2019;24(3):897-904.

5. Czystowska-Kuzmicz M, Whiteside TL. The potential role of tumor-derived exosomes in diagnosis, prognosis, and response to therapy in cancer. Expert Opin Biol Ther. 2020;p. 118. PMID: 32813990 . Available from: https://doi.org/10.1080/ 14712598.2020 .1813276

6. Liu S, et al. Exosomes as critical mediators of cell-to-cell communication in cancer pathogenesis and their potential clinical application. Translational Cancer Research. 2019;8(1):298311. Available from: https://doi.org/10.21037/tcr.2019.01.03.

7. Lotvall J, Valadi H. Cell to cell signalling via exosomes through esRNA. Cell Adh Migr. 2007;1(3):156-158. PMID: 19262134. Available from: https://doi.org/10.4161/cam.1.3.5114.

8. Roma-Rodrigues C, Fernandes AR, Baptista PV. Exosome in tumour microenvironment: overview of the crosstalk between normal and cancer cells. Biomed Res Int. 2014;2014:179486. PMID: 24963475. Available from: https://doi.org/10.1155/ 2014/179486.

9. Meehan B, Rak J, Vizio D. Oncosomes - large and small: what are they, where they came from? J Extracell Vesicles. 2016;5:33109. PMID: 27680302. Available from: https://doi. org/10.3402/jev.v5.33109.

10. Minciacchi VR, et al. MYC Mediates Large Oncosome-Induced Fibroblast Reprogramming in Prostate Cancer. Cancer Res. 2017;77(9):2306-2317. PMID: 28202510. Available from: https://doi.org/10.1158/0008-5472.CAN-16-2942. 
11. Vizio DD, et al. Oncosome formation in prostate cancer: association with a region of frequent chromosomal deletion in metastatic disease. Cancer Res. 2009;69(13):5601-5609. PMID: 19549916. Available from: https://doi.org/10.1158/0008-5472. CAN-08-3860.

12. Osaki M, Okada F. Exosomes and Their Role in Cancer Progression. Yonago Acta Med. 2019;62(2):182-190. PMID: 31320822 Available from: ttps://doi.org/10.33160/yam.2019.06.002.

13. Maia J, et al. Exosome-Based Cell-Cell Communication in the Tumor Microenvironment. Front Cell Dev Biol. 2018;6:18. PMID: 29515996. Available from: https://doi.org/10.3389/fcell. 2018.00018.

14. Qu JL, et al. Gastric cancer exosomes promote tumour cell proliferation through PI3K/Akt and MAPK/ERK activation. Dig Liver Dis. 2009;41(12):875-880. PMID: 19473897. Available from: https://doi.org/10.1016/j.dld.2009.04.006.

15. Pan $\mathrm{L}$, et al. Exosomes-mediated transfer of long noncoding RNA ZFAS1 promotes gastric cancer progression. J Cancer Res Clin Oncol. 2017;143(6):991-1004. PMID: 28285404. Available from: https://doi.org/10.1007/s00432-017-2361-2.

16. Ramteke A, et al. Exosomes secreted under hypoxia enhance invasiveness and stemness of prostate cancer cells by targeting adherens junction molecules. Mol Carcinog. 2015;54(7):554-565. PMID: 24347249. Available from: https: //doi.org/10.1002/mc.22124.

17. Qin X, et al. Cisplatin-resistant lung cancer cell-derived exosomes increase cisplatin resistance of recipient cells in exosomal miR-100-5p-dependent manner. Int J Nanomedicine. 2017;12:3721-3733. PMID: 28553110. Available from: https: //doi.org/10.2147/IJN.S131516.

18. Bu L, et al. Biological heterogeneity and versatility of cancerassociated fibroblasts in the tumor microenvironment. Oncogene. 2019;38(25):4887-4901. PMID: 30816343. Available from: https://doi.org/10.1038/s41388-019-0765-y.

19. Garin-Chesa P, Old LJ, Rettig WJ. Cell surface glycoprotein of reactive stromal fibroblasts as a potential antibody target in human epithelial cancers. Proc Natl Acad Sci U S A. 1990;87(18):7235-7239. PMID: 2402505. Available from: https://doi.org/10.1073/pnas.87.18.7235.

20. Arandkar S, et al. Altered p53 functionality in cancerassociated fibroblasts contributes to their cancer-supporting features. Proc Natl Acad Sci U S A. 2018;115(25):6410-6415. PMID: 29866855. Available from: https://doi.org/10.1073/pnas. 1719076115.

21. Cheteh $\mathrm{EH}$, et al. Interleukin- 6 derived from cancer-associated fibroblasts attenuates the p53 response to doxorubicin in prostate cancer cells. Cell Death Discov. 2020;6:42. PMID: 32528731. Available from: https://doi.org/10.1038/s41420020-0272-5.

22. Kalluri R, Zeisberg M. Fibroblasts in cancer. Nat Rev Cancer. 2006;6(5):392-401. PMID: 16572188.

23. Karnoub $A E$, et al. Mesenchymal stem cells within tumour stroma promote breast cancer metastasis. Nature. 2007;449(7162):557-563. PMID: 17914389. Available from: https://doi.org/10.1038/nature06188.

24. Zhang $Y$, et al. Stromal progenitor cells from endogenous adipose tissue contribute to pericytes and adipocytes that populate the tumor microenvironment. Cancer Res. 2012;72(20):5198-5208. PMID: 23071132. Available from: https://doi.org/10.1158/0008-5472.CAN-12-0294.

25. Direkze NC, et al. Bone marrow contribution to tumorassociated myofibroblasts and fibroblasts. Cancer Res. 2004;64(23):8492-8495. PMID: 15574751.

26. Wilde $\mathrm{L}$, et al. Metabolic coupling and the Reverse Warburg Effect in cancer: Implications for novel biomarker and anticancer agent development. Semin Oncol. 2017;44(3):198203. PMID: 29248131. Available from: https://doi.org/10.1053/ j.seminoncol.2017.10.004

27. Fong MY, et al. Breast-cancer-secreted miR-122 reprograms glucose metabolism in premetastatic niche to promote metastasis. Nat Cell Biol. 2015;17(2):183-194. PMID:
25621950. Available from: https://doi.org/10.1038/ncb3094.

28. Webber J, et al. Cancer exosomes trigger fibroblast to myofibroblast differentiation. Cancer Res. 2010;70(23):9621-9630. PMID: 21098712. Available from: https://doi.org/10.1158/ 0008-5472.CAN-10- 1722.

29. Yan L, et al. Cancer-associated fibroblasts-derived exosomesmediated transfer of LINC00355 regulates bladder cancer cell proliferation and invasion. Cell Biochem Funct. 2020;38(3):257-265. PMID: 31749189. Available from: https: //doi.org/10.1002/cbf.3462.

30. Lee AH, et al. Ovarian Cancer Exosomes Trigger Differential Biophysical Response in Tumor-Derived Fibroblasts. Sci Rep. 2020;(1):8686. PMID: 32457479. Available from: https://doi. org/10.1038/s41598-020-65628-3.

31. Richards DM, Hettinger J, Feuerer M. Monocytes and macrophages in cancer: development and functions. Cancer Microenviron. 2013;6(2):179-191. PMID: 23179263. Available from: https://doi.org/10.1007/s12307-012-0123-x.

32. Hu W, et al. Cancer Immunotherapy Based on Natural Killer Cells: Current Progress and New Opportunities. 2019;10:1205. PMID: 31214177. Available from: https://doi.org/10.3389/ fimmu.2019.01205.

33. Ribeiro MF, et al. Exosomes Function in Pro- and AntiAngiogenesis. Curr Angiogenes. 2013;2(1):54-59.

34. Taverna $S$, et al. Role of exosomes released by chronic myelogenous leukemia cells in angiogenesis. Int J Cancer. 2012;130(9):2033-2043. PMID: 21630268. Available from: https://doi.org/10.1002/ijc.26217.

35. Thompson CA, et al. Heparanase regulates secretion, composition, and function of tumor cell-derived exosomes. J Biol Chem. 2013;288(14):10093-10099. PMID: 23430739. Available from: https://doi.org/10.1074/jbc.C112.444562.

36. Qiu JJ, et al. Exosomal MetastasisAssociated Lung Adenocarcinoma Transcript 1 Promotes Angiogenesis and Predicts Poor Prognosis in Epithelial Ovarian Cancer. Int J Biol Sci. 2018;14(14):1960-1973. PMID: 30585260. Available from: https://doi.org/10.7150/ijbs.28048.

37. Zhou M, et al. Pancreatic cancer derived exosomes regulate the expression of TLR4 in dendritic cells via miR-203. Cell Immunol. 2014;292(1-2):65-69. PMID: 25290620. Available from: https://doi.org/10.1016/j.cellimm.2014.09.004.

38. Treps $L$, et al. Glioblastoma stem-like cells secrete the proangiogenic VEGF-A factor in extracellular vesicles. J Extracell Vesicles. 2017;6(1):1359479. PMID: 28815003. Available from: https://doi.org/10.1080/20013078.2017.1359479.

39. Duan $\mathrm{P}$, et al. Potential role of exosomes in the pathophysiology, diagnosis, and treatment of hypoxic diseases. Am J Transl Res. 2019;11(3):1184-1201.

40. Kucharzewska $P$, et al. Exosomes reflect the hypoxic status of glioma cells and mediate hypoxia-dependent activation of vascular cells during tumor development. Proc Natl Acad Sci U S A. 2013;110(18):7312-7317. PMID: 23589885. Available from: https://doi.org/10.1073/pnas.1220998110.

41. Huang Z, Feng Y. Exosomes Derived From Hypoxic Colorectal Cancer Cells Promote Angiogenesis Through Wnt4Induced beta-Catenin Signaling in Endothelial Cells. Oncol Res. 2017;25(5):651-661. PMID: 27712599. Available from: https://doi.org/10.3727/096504016X14752792816791.

42. Lai RC, et al. Proteolytic Potential of the MSC Exosome Proteome: Implications for an Exosome-Mediated Delivery of Therapeutic Proteasome. Int J Proteomics. 2012;2012:971907. PMID: 22852084. Available from: https://doi.org/10.1155/ 2012/971907.

43. Phan J, et al. Engineering mesenchymal stem cells to improve their exosome efficacy and yield for cell-free therapy. J Extracell Vesicles. 2018;7(1):1522236. PMID: 30275938. Available from: https://doi.org/10.1080/20013078.2018.1522236.

44. Reza-Zaldivar EE, et al. Potential Effects of MSC-Derived Exosomes in Neuroplasticity in Alzheimer's Disease. Front Cell Neurosci. 2018;12:317. PMID: 30319358. Available from: https: //doi.org/10.3389/fncel.2018.00317. 
45. Yin K, Wang S, Zhao RC. Exosomes from mesenchymal stem/stromal cells: a new therapeutic paradigm. Biomark Res. 2019;7:8. PMID: 30992990. Available from: https://doi.org/10. 1186/s40364-019-0159-x.

46. Zhu W, Chen H, Wang J. Engineered/Hypoxia-Preconditioned MSC-Derived Exosome: Its Potential Therapeutic Applications, in Mesenchymal Stem Cell Derived Exosomes: The Potential for Translational Nanomedicine. Y. Tang and B. Dawn, Editors. Academic Press. 2015;p. 139-159. Available from: https://doi.org/10.1016/B978-0-12-800164-6.00007-1.

47. Wang $S$, et al. Exosomes secreted by mesenchymal stro$\mathrm{mal} / \mathrm{stem}$ cell-derived adipocytes promote breast cancer cell growth via activation of Hippo signaling pathway. Stem Cell Res Ther. 2019;10(1):117. PMID: 30971292. Available from: https://doi.org/10.1186/s13287-019-1220-2.

48. Zhou J, et al. Mesenchymal Stem Cell Derived Exosomes in Cancer Progression, Metastasis and Drug Delivery: A Comprehensive Review. J Cancer. 2018;9(17):3129-3137. PMID: 30210636. Available from: https://doi.org/10.7150/jca.25376.

49. Karaoz E, Sun E, Demir CS. Mesenchymal stem cell-derived exosomes do not promote the proliferation of cancer cells in vitro. Int J Physiol Pathophysiol Pharmacol. 2019;11(4):177189.

50. Rosenberger $L$, et al. Stem cell exosomes inhibit angiogenesis and tumor growth of oral squamous cell carcinoma. Sci Rep. 2019;9(1):663. PMID: 30679544. Available from: https://doi. org/10.1038/s41598-018-36855-6.

51. Bruno $S$, et al. Microvesicles derived from human bone marrow mesenchymal stem cells inhibit tumor growth. Stem Cells Dev. 2013;22(5):758-771. PMID: 23034046. Available from: https://doi.org/10.1089/scd.2012.0304.

52. Ko SF, et al. Adipose-Derived Mesenchymal Stem Cell Exosomes Suppress Hepatocellular Carcinoma Growth in a Rat Model: Apparent Diffusion Coefficient, Natural Killer T-Cell Responses, and Histopathological Features. Stem Cells Int. 2015;2015:853506. PMID: 26345219. Available from: https: //doi.org/10.1155/2015/853506.

53. Alzahrani FA, et al. Potential Effect of Exosomes Derived from Cancer Stem Cells and MSCs on Progression of DENInduced HCC in Rats. Stem Cells Int. 2018;2018:8058979. PMID: 30224923. Available from: https://doi.org/10.1155/ 2018/8058979.

54. Hanahan $D$, Weinberg RA. Hallmarks of cancer: the next generation. Cell. 2011;144(5):646-674. PMID: 21376230. Available from: https://doi.org/10.1016/j.cell.2011.02.013.

55. Czernek L, Duchler M. Functions of Cancer-Derived Extracellular Vesicles in Immunosuppression. Arch Immunol Ther Exp (Warsz). 2017;65(4):311-323. PMID:28101591. Available from: https://doi.org/10.1007/s00005-016-0453-3.

56. Haderk $F$, et al. Tumor-derived exosomes modulate PD-L1 expression in monocytes. Sci Immunol. 2017;2(13). PMID: 28754746. Available from: https://doi.org/10.1126/ sciimmunol.aah5509.

57. Olejarz W, et al. Tumor-Derived Exosomes in Immunosuppression and Immunotherapy. J Immunol Res. 2020;2020:6272498. PMID: 32537468. Available from: https://doi.org/10.1155/2020/6272498.

58. Nichols BA, Bainton DF, Farquhar MG. Differentiation of monocytes. Origin, nature, and fate of their azurophil granules. J Cell Biol. 1971;50(2):498-515. PMID: 4107019. Available from: https://doi.org/10.1083/jcb.50.2.498.

59. Sampath $\mathrm{P}$, et al. Monocyte Subsets: Phenotypes and Function in Tuberculosis Infection. Front Immunol. 2018;9:1726. PMID: 30105020 . Available from: https://doi.org/10.3389/ fimmu.2018.01726.

60. Shi C, Pamer EG. Monocyte recruitment during infection and inflammation. Nat Rev Immunol. 2011;11(11):762-774. PMID: 21984070. Available from: https://doi.org/10.1038/nri3070.

61. Yang J, et al. Monocyte and macrophage differentiation: circulation inflammatory monocyte as biomarker for inflammatory diseases. Biomark Res. 2014;2(1):1. PMID: 24398220. Available from: https://doi.org/10.1186/2050-7771-2-1.
62. Atretkhany KN, et al. Chemokines, cytokines and exosomes help tumors to shape inflammatory microenvironment. Pharmacol Ther. 2016;168:98-112. PMID: 27613100. Available from: https://doi.org/10.1016/j.pharmthera.2016.09.011.

63. Zamarron BF, Chen W. Dual roles of immune cells and their factors in cancer development and progression. Int J Biol Sci. 2011;7(5):651-658. PMID: 21647333. Available from: https: //doi.org/10.7150/ijbs.7.651.

64. Karin M, et al. NF-kappaB in cancer: from innocent bystander to major culprit. Nat Rev Cancer. 2002;2(4):301-310. PMID: 12001991. Available from: https://doi.org/10.1038/nrc780.

65. Bretz NP, et al. Body fluid exosomes promote secretion of inflammatory cytokines in monocytic cells via Toll-like receptor signaling. J Biol Chem. 2013;288(51):36691-36702. PMID: 24225954. Available from: https://doi.org/10.1074/jbc.M113. 512806 .

66. Grivennikov SI, Karin M. Dangerous liaisons: STAT3 and NF-kappaB collaboration and crosstalk in cancer. Cytokine Growth Factor Rev. 2010;21(1):11-19. PMID: 20018552. Available from: https://doi.org/10.1016/j.cytogfr.2009.11.005.

67. Martinez FO, et al. Macrophage activation and polarization Front Biosci. 2008;13:453-461. PMID: 17981560. Available from: https://doi.org/10.2741/2692.

68. Othman N, Jamal R, Abu N. Cancer-Derived Exosomes as Effectors of Key Inflammation-Related Players. Front Immunol. 2019;10:2103. PMID: 31555295. Available from: https://doi. org/10.3389/fimmu.2019.02103.

69. Chen Z, et al. Cytoskeleton-centric protein transportation by exosomes transforms tumor-favorable macrophages. Oncotarget. 2016;7(41):67387-67402. PMID: 27602764. Available from: https://doi.org/10.18632/oncotarget.11794.

70. Chow, A., et al., Macrophage immunomodulation by breast cancer-derived exosomes requires Toll-like receptor 2-mediated activation of NF-kappaB. Sci Rep. 2014;4:5750. PMID: 25034888. Available from: https://doi.org/10.1038/srep05750.

71. Howard CJ, et al. The role of dendritic cells in shaping the immune response. Anim Health Res Rev. 2004;5(2):191-195. PMID: 15984324. Available from: https://doi.org/10.1079/ AHR200468.

72. Yang L, Carbone DP. Tumor-host immune interactions and dendritic cell dysfunction. Adv Cancer Res. 2004;92:1327. Available from: https://doi.org/10.1016/S0065-230X(04) 92002-7.

73. Yu S, et al. Tumor exosomes inhibit differentiation of bone marrow dendritic cells. J Immunol. 2007;178(11):6867-6875. PMID: 17513735. Available from: https://doi.org/10.4049/ jimmunol.178.11.6867.

74. Gabrilovich DI. Myeloid-Derived Suppressor Cells. Cancer Immunol Res. 2017;5(1):3-8. PMID: 28052991. Available from: https://doi.org/10.1158/2326-6066.CIR-16-0297.

75. Kumar V, et al. The Nature of Myeloid-Derived Suppressor Cells in the Tumor Microenvironment. Trends Immunol. 2016;37(3):208-220. PMID: 26858199. Available from: https: //doi.org/10.1016/j.it.2016.01.004

76. Xiang X, et al. Induction of myeloid-derived suppressor cells by tumor exosomes. Int J Cancer. PMid:. 2009;124(11):26212633. PMID: 19235923. Available from: https://doi.org/10. 1002/ijc.24249.

77. Chalmin F, et al. Membrane-associated Hsp72 from tumorderived exosomes mediates STAT3-dependent immunosuppressive function of mouse and human myeloid-derived suppressor cells. J Clin Invest. 2010;120(2):457-471. PMID: 20093776. Available from: https://doi.org/10.1172/JCI40483.

78. Diao J, et al. Exosomal Hsp70 mediates immunosuppressive activity of the myeloid-derived suppressor cells via phosphorylation of Stat3. Med Oncol. 2015;32(2):453. PMID: 25603952. Available from: https://doi.org/10.1007/s12032-014-0453-2.

79. $\mathrm{Ni} \mathrm{C}$, et al. Breast cancer-derived exosomes transmit IncRNA SNHG16 to induce CD73+gammadelta1 Treg cells. Signal Transduct Target Ther. 2020;5(1):41. PMID: 32345959. Avail- 
able from: https://doi.org/10.1038/s41392-020-0129-7.

80. Chen $\mathrm{X}$, et al. Exosomes derived from hypoxic epithelial ovarian cancer deliver microRNA-940 to induce macrophage M2 polarization. Oncol Rep. 2017;38(1):522-528. PMID: 28586039. Available from: https://doi.org/10.3892/or.2017. 5697.

81. Liang ZX, et al. LncRNA RPPH1 promotes colorectal cancer metastasis by interacting with TUBB3 and by promoting exosomes-mediated macrophage M2 polarization. Cell Death Dis. 2019;10(11):829. PMID: 31685807. Available from: https: //doi.org/10.1038/s41419-019-2077-0.

82. Mantovani A, Sica A. Macrophages, innate immunity and cancer: balance, tolerance, and diversity. Curr Opin Immunol. 2010;22(2):231-237. PMID: 20144856. Available from: https: //doi.org/10.1016/j.coi.2010.01.009.

83. Chen G, et al. Exosomal PD-L1 contributes to immunosuppression and is associated with anti-PD-1 response. Nature. 2018;560(7718):382-386. PMID: 30089911. Available from: https://doi.org/10.1038/s41586-018-0392-8.

84. Sharma $\mathrm{P}$, et al. Melanoma cell-derived exosomes in plasma of melanoma patients suppress functions of immune effector cells. Sci Rep. 2020;10(1):92. PMID: 31919420. Available from: https://doi.org/10.1038/s41598-019-56542-4.

85. Liu J, et al. Immune suppressed tumor microenvironment by exosomes derived from gastric cancer cells via modulating immune functions. Sci Rep. 2020;10(1):14749. PMID: 32901082. Available from: https://doi.org/10.1038/s41598020-71573-y.

86. Gabrusiewicz K, et al. Glioblastoma stem cell-derived exosomes induce M2 macrophages and PD-L1 expression on human monocytes. Oncoimmunology. 2018;7(4):e1412909. PMID: 29632728. Available from: https://doi.org/10.1080/ 2162402X.2017.1412909.

87. Anastasiadou E, Slack FJ. Cancer. Malicious exosomes. Science. 2014;346(6216):1459-1460. PMID: 25525233. Available from: https://doi.org/10.1126/science.aaa4024.
88. Xie $\mathrm{F}$, et al. Extracellular Vesicles in Cancer Immune Microenvironment and Cancer Immunotherapy. Adv Sci (Weinh). 2019;6(24):1901779. Available from: https://doi.org/10.1002/ advs.201901779.

89. Chatterjee SK, Zetter BR. Cancer biomarkers: knowing the present and predicting the future. Future Oncol. 2005;1(1):3750. PMID: 16555974. Available from: https://doi.org/10.1517/ 14796694.1.1.37.

90. Kalra $\mathrm{H}$, et al. Comparative proteomics evaluation of plasma exosome isolation techniques and assessment of the stability of exosomes in normal human blood plasma. Proteomics. 2013;13(22):3354-3364. PMID: 24115447. Available from: https://doi.org/10.1002/pmic.201300282.

91. Muller $\mathrm{L}$, et al. Isolation of biologically-active exosomes from human plasma. J Immunol Methods. 2014;411:55-65. PMID: 24952243. Available from: https://doi.org/10.1016/j.jim.2014. 06.007 .

92. Hoshino $A$, et al. Tumour exosome integrins determine organotropic metastasis. Nature. 2015;527(7578):329-335 PMID: 26524530. Available from: https://doi.org/10.1038/ nature 15756.

93. Peinado $\mathrm{H}$, et al. Melanoma exosomes educate bone marrow progenitor cells toward a pro-metastatic phenotype through MET. Nat Med. 2012;18(6):883-891. PMID: 22635005. Available from: https://doi.org/10.1038/nm.2753.

94. Hofmann L, et al. The Emerging Role of Exosomes in Diagnosis, Prognosis, and Therapy in Head and Neck Cancer Int J Mol Sci. 2020;21(11). PMID: 32517240. Available from https://doi.org/10.3390/ijms21114072.

95. Melo SA et al. Glypican-1 identifies cancer exosomes and detects early pancreatic cancer. Nature. 2015;523(7559):177182. PMID: 26106858. Available from: https://doi.org/10.1038/ nature14581.

96. Sheridan C. Exosome cancer diagnostic reaches market. Nat Biotechnol. 2016:34(4):359-360. PMID: 27054974. Available from: https://doi.org/10.1038/nbt0416-359. 